\title{
CIGRE-Session 2012
}

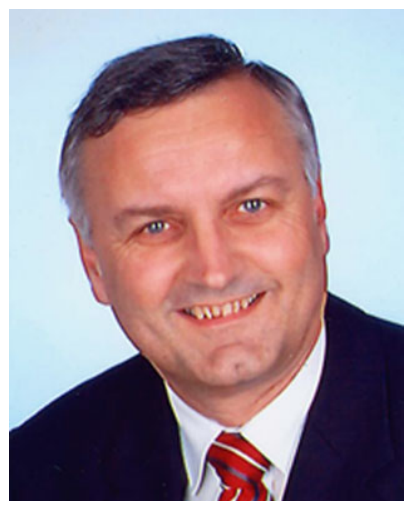

Dipl.-Ing. Karl Bauer
Die 44. CIGRE-Session fand vom 26. bis 31. August 2012 in Paris statt. Mit 3200 Teilnehmern, darunter 58 aus Österreich, aus 90 Ländern und 3626 Besuchern an der parallel zur CIGRE-Session stattfindenden technischen Ausstellung wurden sämtliche Teilnehmer- und Besucherzahlen der früheren Sessionen weit übertroffen.

Die technische Ausstellung konnte durch die Aufteilung auf zwei Ebenen um $50 \%$ auf eine Ausstellungsfläche von $8560 \mathrm{~m}^{2}$ erweitert werden. Die Anzahl der Aussteller stieg auf 198.

Die CIGRE-Session ist eines der weltweit wichtigsten Zusammentreffen von Managern, Ingenieuren und Experten aus der Elektrotechnik, den Bereichen Forschung und Lehre, Energiewirtschaft und Industrie. Die Konferenz bietet eine einzigartige Gelegenheit zur Vernetzung und zum Wissensaustausch.

Folgende Themen wurden $u$. a. behandelt und diskutiert:

- Eco design of equipment

- Maintenance, refurbishment and life time of equipment

- Real time monitoring of equipment and systems

- Development of DC solutions

- Coordination between TSOs

- Public acceptance of equipment

- Integration of renewable
- Active distribution systems

- Information systems

Der elektrische Energiesektor und das elektrische Netz sind heute einem starken dynamischen Wandel ausgesetzt. Für die CIGRE bedeutet das eine Erweiterung ihres technischen Verantwortungsbereiches. Sie behandelt daher - neben den angestammten Übertragungsthemen - verstärkt die Probleme im Verteilnetz, wie das "Smart Grid".

Abgestimmt auf die 16 Studienkomitees der CIGRE wurden aus den vielen eingereichten Beiträgen über 400 ausgewählt und in den einzelnen Plenar- oder Poster-Sessionen präsentiert und diskutiert. Acht Beiträge, die Sie auf den folgenden Seiten nachlesen können, kamen aus Österreich.

Im Rahmen der Konferenz fanden auch die Vollversammlung, die Sitzungen des Administrative Council und einzelner Nationalkomitees sowie die Neuwahl der Verwaltungsorgane statt. Beim Vorsitzenden des technischen Komitees fiel die Wahl auf Mark Waldron, National Grid, Großbritannien.

Zum Präsidenten der CIGRE wählte das Administrative Council aus drei Kandidaten Prof. Dr. Klaus Fröhlich. Fröhlich wurde 1990 zum Professor an der TU Wien berufen. Ab 1997 leitete er als Professor die Fachgruppe Hochspannungstechnologie an der ETH Zürich. Bereits als Präsident des technischen Komitees der CIGRE hat sich Fröhlich für eine nachhaltige Energieversorgung eingesetzt. Das Österreichische Nationalkomitee gratuliert herzlich zu dieser hohen Ehre!

Im Folgenden finden Sie weiters die Berichte über die Diskussionen der Studienkomitees der CIGRE.

Eine angenehme Lektüre wünscht Ihnen

Dipl.-Ing. Karl Bauer OVE

Vorsitzender des Österreichischen Nationalkomitees der CIGRE 